\title{
Estafiloma peripapilar, presentación clínica y diagnósticos diferenciales en anomalías congénitas de nervio óptico
}

\author{
Peripapillary staphyloma, clinical presentation, and differential diagnoses in \\ congenital optic nerve anomalies \\ Matías Bagolini ${ }^{*}$, Cristián Salgado
}

\section{Resumen}

Un paciente de 3 meses con sospecha de estrabismo presenta al examen una gran anisometropía con -9.50 esfera en su ojo izquierdo y al fondo de ojo destaca una papila alterada similar a la papila de Morning-Glory. Las anomalías congénitas de nervio óptico (ACNO) son un grupo de condiciones que se presentan clínicamente como baja agudeza visual, estrabismo y/o nistagmus. El diagnóstico diferencial es clínico y complejo por la sobre posición morfológica entre estas. Los principales diagnósticos diferenciales son la papila de Morning-Glory, el coloboma y la hipoplasia de nervio óptico. Se recomienda manejo multidisciplinario por sus asociaciones sistémicas.

Palabras clave: nervio óptico; coloboma; Morning-Glory; anomalía congénita; estafiloma peripapilar; hipoplasia nervio óptico.

\begin{abstract}
A 3-month-old patient with a suspicion of strabismus on examination has a great anisometropia with -9.50 sphere in his left eye and at the fundus examination an altered papilla similar to the Morning-Glory papilla. Congenital optic nerve abnormalities (ANOC) are a group of conditions that present clinically as low visual acuity, strabismus, and nystagmus. The differential diagnosis is clinical and complex due to the morphological position between them. The main differential diagnoses are the Morning-Glory papilla, the coloboma, and optic nerve hypoplasia. Multidisciplinary management is recommended for its systemic associations.
\end{abstract}

Keywords: optic nerve, coloboma; Morning-Glory ; congenital anomaly; peripapillary staphyloma; optic nerve hypoplasia.

Fecha de envío: 16 de abril de 2020 - Fecha de aceptación: 28 de Agosto de 2020

\section{Introducción}

Las anomalías congénitas de nervio óptico (ACNO) son un grupo de condiciones que se presentan clínicamente como baja agudeza visual, estrabismo o nistagmus y requieren una evaluación completa y cuidadosa por su posible asociación a cuadros sistémicos relevantes como, por ejemplo, la vasculopatía arterial progresiva conocida como enfermedad de Moya-Moya. El diagnóstico entre las diferentes causas es principalmente clínico y muchas veces complejo debido al solapamiento morfológico que puede existir. Dentro de las causas más importantes se encuentra la hipoplasia de nervio óptico (HNO), anomalía papilar de Morning-Glory (AMG), coloboma de nervio óptico, estafiloma peripapilar (EP) y drusas de nervio óptico (Capo et al., 2011). Cada una de ellas conlleva una actitud terapéutica diferente y un pronóstico potencialmente grave si no se realiza el diagnóstico correcto. En el Reino Unido éstas son causa de hasta un $15 \%$ de los casos de baja visual severa o ceguera en población pediátrica (Taylor, 2007).

El estafiloma peripapilar es una ACNO no hereditaria, unilateral en dónde el nervio óptico se encuentra inmerso en una gran ectasia del polo posterior, pudiéndose encontrar una papila que presenta una vasculatura radial y tejido glial central. Es frecuente su asociación con alta miopía y los pacientes deben ser seguidos por el riesgo aumentado de desarrollar desprendimientos serosos de retina alrededor del nervio óptico (Kim, 2005).

\section{Reporte de caso}

Se presenta el caso de un paciente de 3 meses, que consulta por sospecha de estrabismo. Al examen el paciente presenta fijación y 
seguimiento fugaz con ambos ojos, la exploración sensoriomotora revela una endotropia (ET) izquierda con ángulo que varía entre $5^{\circ}$ y $15^{\circ}$ al test de Hirschberg, con nistagmus monocular izquierdo tipo resorte intermitente en posición primaria de mirada, que aumenta en las lateroversiones. Llama la atención una fuerte preferencia fijación del ojo derecho y a la refracción con gotas dilatadoras (esquiascopía) se constata una gran anisometropía de +3.00 esf en su ojo derecho (OD), y -9,50 esf en su ojo izquierdo (OI).

Al fondo de ojo destaca: OD normal y OI papila rosada neta incluida en un gran estafiloma posterior con vasculatura anormal, mácula de aspecto normal. El polo anterior y el resto del examen es normal (Figura 1).

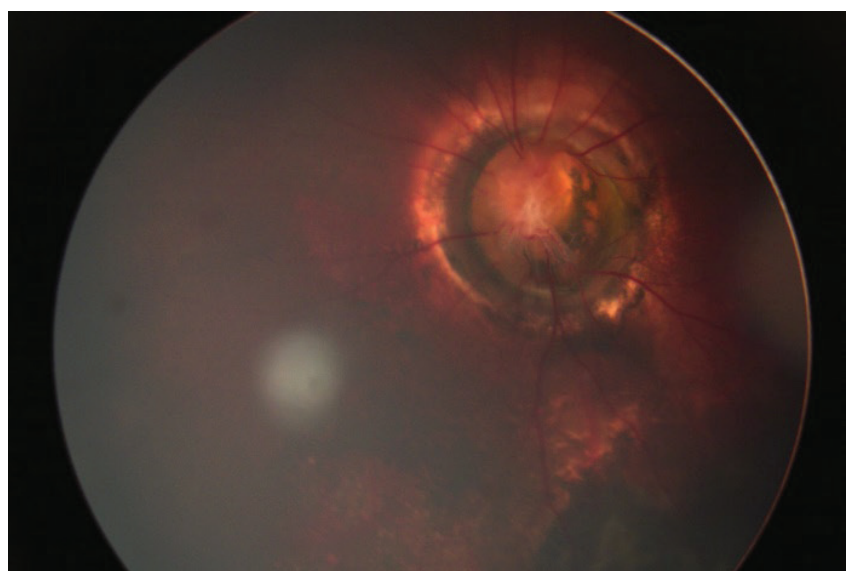

Figura 1: Foto del nervio óptico. Destaca una emergencia radial de la vasculatura, asociado a una papila grisácea, sin coloboma.

Con sospecha diagnóstica de ACNO unilateral se decide realizar un examen bajo anestesia general para caracterizar mejor los hallazgos encontrados al examen clínico, en el cual destaca un largo axial aumentado en OI (20,12 mm) en comparación con OD $(18,77 \mathrm{~mm})$, sin diferencias en presión intraocular, paquimetría y diámetro corneal.

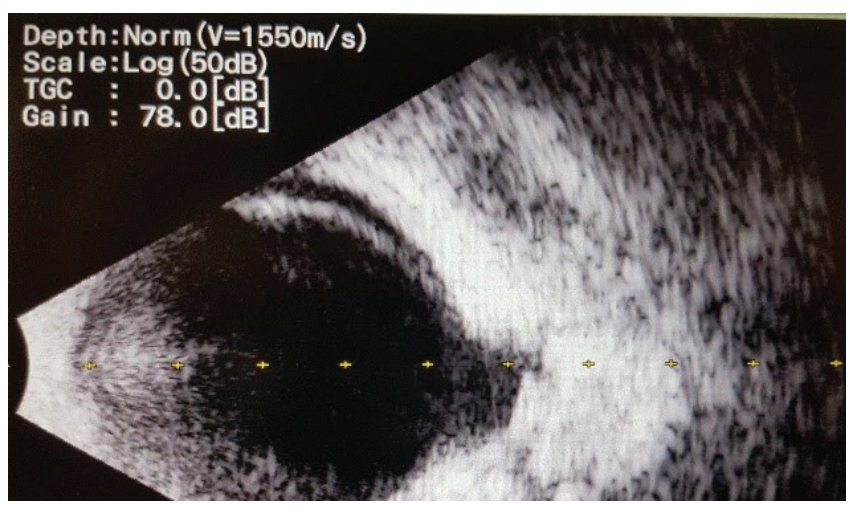

Figura 2: Ecografía modo B. destaca un gran estafiloma en polo posterior que incluye al nervio óptico.
Considerando el estafiloma detectado en la ecografía, se define la ACNO del OI del paciente como un estafiloma peripapilar asociado a alta miopía. En este contexto, se inicia manejo con corrección óptica guiada por esquiascopía y parche en el OD 4 horas al día.

En el seguimiento posterior con test de teller (al año de tratamiento), el paciente presentaba una AV normal en su OD y mejoría significativa de su AV en OI con teller que mejoró de 0,86 cy/cm a $1.3 \mathrm{cy} / \mathrm{cm}$ en 1 año, sin embargo, aún bajo lo normal para su edad. Actualmente, el paciente se mantiene en tratamiento con lentes ópticos, parche ocular y presenta además una ET izquierda de $15^{\circ}$ con fuerte preferencia fijación en su OD, la cual se resolverá quirúrgicamente.

\section{Discusión}

Las anomalías congénitas de nervio óptico son un grupo de condiciones que se pueden presentar clínicamente como baja agudeza visual, estrabismo o nistagmus (Capo et al., 2011). La subclasificación de las diferentes ACNO ha permitido asociar morfológicamente las papilas anómalas con posibles malformaciones del sistema nervioso central. Esta distinción es particularmente compleja debido a la gran superposición morfológica existente entre papilas malformadas congénitamente (Brodsky, 1994). Sin embargo, ha habido avances en la utilización de la tomografía de coherencia óptica (OCT), identificando características propias de cada patología, lo que permite un diagnóstico más certero en casos complejos, y el seguimiento de posibles complicaciones como membrana neovascular coroídea (MNVC). (Jeng-Miller 2017)

En las ACNO, a diferencia de lo que ocurre en la mayoría de las neuropatías ópticas adquiridas, la visión de colores es en general normal.

A continuación, se discutirán las características principales de los distintos tipos de ACNO, que permiten realizar un adecuado diagnóstico diferencial y su consecuente estudio y/o manejo.

\section{Hipoplasia de nervio óptico}

Esta alteración puede pasar desapercibida si no se presta especial atención al examen de la papila, es la ACNO más frecuente y se asocia al consumo de alcohol y drogas durante el embarazo (Brodsky, 1991). Oftalmoscópicamente se presenta como un nervio óptico pequeño gris o pálido y que puede asociarse a un anillo peripapilar hiper o hipopigmentado (signo del doble anillo) difícil de distinguir del borde neuroretinal. Clásicamente se debe sospechar esta patología en pacientes que tengan una distancia mayor a 2 diámetros discales del centro del NO a la mácula (Pollock, 1987). Estos cambios también pueden ser objetivados al 
OCT, en donde encontraremos un menor diámetro discal asociado a disminución en el grosor de las capas retinales tanto internas como externas, incluyendo a la fóvea (Jeng-Miller 2017).

Los pacientes con HNO tienen constricción en su campo visual y la agudeza visual puede variar entre $20 / 20$ y visión cero, no teniendo relación con el tamaño del NO. Hay que estar atentos a defectos refractivos como el astigmatismo, frecuentemente asociados (Weiss \& Kelly, 2003).

La HNO puede estar asociada a malformaciones del sistema nervioso central (SNC) como la displasia septo-óptica (Sd. Mosier) que característicamente presenta pequeñas malformaciones a nivel de vía visual anterior como la ausencia de septum pelúcidum junto a la agenesia del cuerpo calloso y disminución en la producción de la hormona del crecimiento, sin embargo, puede estar alterada la producción de cualquiera de las hormonas hipofisiarias (De Morsier, 1956). Un estudio reciente demostró que la HNO bilateral se asocia más frecuentemente a retraso en el desarrollo psicomotor que la HNO unilateral (Garcia-Filion, 2010). Es por esta razón que todos los pacientes deben ser estudiados con resonancia nuclear magnética del SNC (la cual debe ser solicitada con cortes de vía visual, recordando que también puede estar hipoplásica) y estudio endocrinológico que incluya estudio de hormonas hipofisiarias, especialmente la hormona del crecimiento (Brodsky, 1990).

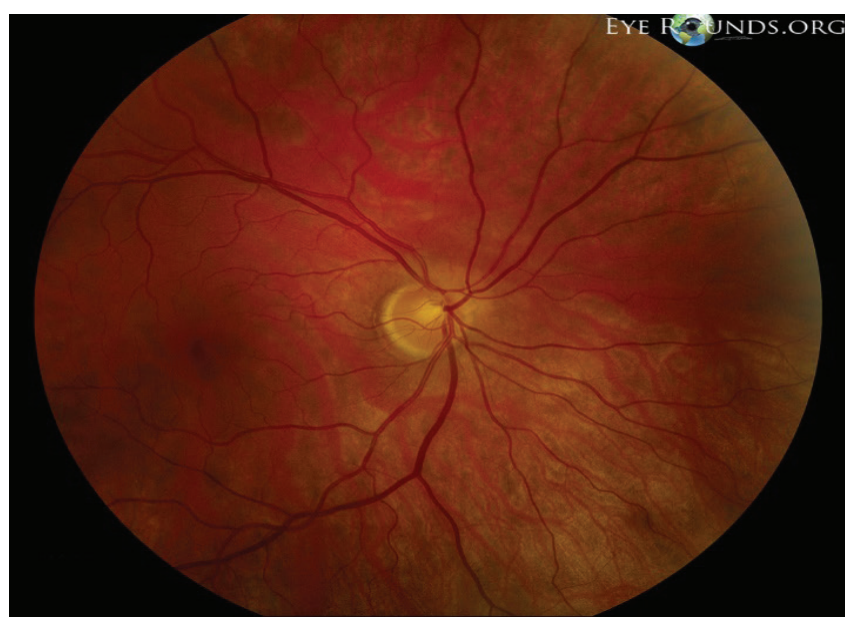

Figura 3: Hipoplasia de nervio óptico. Se observa la presencia de una distancia mayor a 2 diámetros discales desde el centro del NO a la fóvea (Stem Cell Care India, 2019).

\section{Coloboma de nervio óptico}

El término coloboma, proveniente del griego, significa truncado o mutilado y es en esencia como se ve, una papila grande y alargada con una excavación bien delimitada en forma "de tazón" blanco, que se extiende anormalmente hacia inferior. El borde neurorretinal de la papila es típicamente adelgazado a inferior y normal o engrosado a superior (Brodsky, 1994). El coloboma de NO puede ser unilateral o bilateral en igual frecuencia, y puede asociarse a coloboma de párpado, iris o incluso retina y coroides dentro de su presentación clínica. La agudeza visual no se correlaciona con la magnitud del coloboma y es variable, dependiendo principalmente de la afectación del haz papilomacular, lo cual es clínicamente muy difícil de anticipar (Pollock, 1987). Pese a que no es indispensable en su diagnóstico, el OCT nos puede ayudar a detectar una MNVC, la cual podría estar asociada a esta patología (Jeng-Miller 2017).

Dentro de los síndromes asociados están el CHARGE (Del inglés, coloboma, heart defects, atresia choanae, growth retardation, genital abnormalities, and ear abnormalities), el síndrome de Aicardi (que presenta atrofia corioretinal en parches y cataratas), o la secuencia de Goldenhar caracterizada por afecciones oculares (tales como quistes dermoides y lipodermoides en los párpados, dermoide epibulbar, colobomas, entre otros) auriculares (desde anotia a un pabellón auricular dismórfico) y vertebrales. (Brodsky, 1994). Es por esto que el clínico debe buscar dirigidamente otras anomalías al examen oftalmológico, las cuales permitirán detectar tempranamente estas asociaciones.

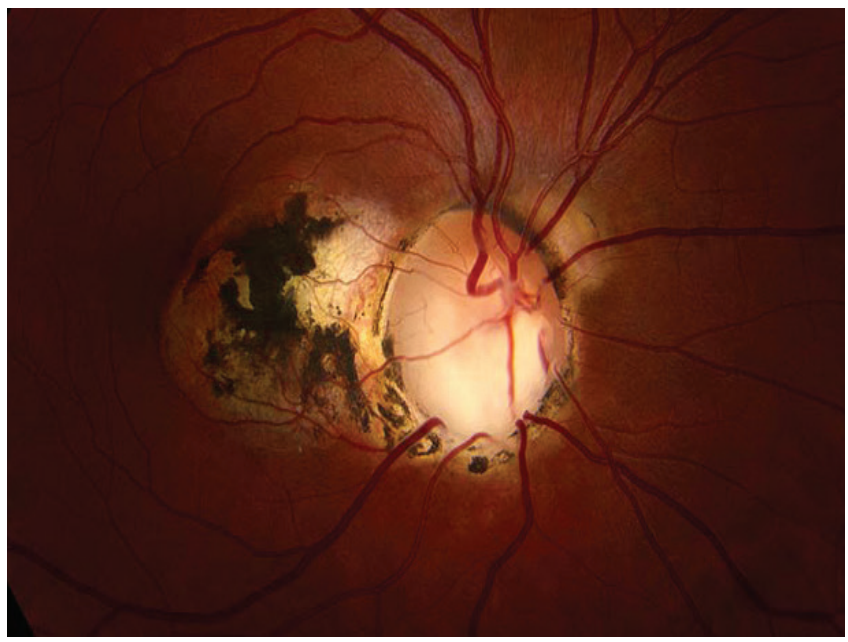

Figura 4: Coloboma de Nervio óptico y MNVC peripapilar en regresión. Aspecto de una papila con diagnóstico de coloboma. Destaca su palidez y excavación grande hacia inferior. Además, este paciente presentó una MNVC peripapilar, que al momento de la imagen ya se encontraba en resolución (Ophthalmic Atlas Images by EyeRounds.org, 2015).

\section{Anomalía de Morning-Glory}

Nombrada así por la similitud con la flor del mismo nombre, es una anomalía congénita en que el nervio óptico presenta una excavación en forma de embudo. A la oftalmoscopía la papíla en la AMG es grande, rosada o levemente anaranjada, con un borde de alteración pigmentaria corioretinal que la rodea por toda su 
periferia (Jonas, 1989). Presenta, además tejido glial central, levemente azulado y aspecto solevantado. Los vasos emergen radialmente, para luego seguir un trayecto recto por la retina, estando muchas veces alterados en morfología y número. La agudeza visual es generalmente pobre, pudiendo ser desde 20/20 a visión cuenta dedos (Pollock, 1987).

Las características al OCT están bien establecidas mostrando un diámetro del nervio óptico aumentado con un escaso borde neuroretinal y un aumento en la capa de fibras nerviosas. En la mácula, por el contrario, el espesor de la capa de fibras está reducido (Jeng-Miller 2017).

La asociación con encefalocele transesfenoidal está bien establecida y clínicamente se puede sospechar por una zona depigmentada en forma de $\mathrm{V}$ (forma de lengua) a inferior de la papila. De presentarse, la encefalocele puede tener complicaciones al momento de la intubación anestésica (Beyer, 1982; Caprioli \& Lesser, 1983).

Con la ayuda de la angio-RNM, se han reportado asociaciones de la AMG con disgenesias vasculares cerebrales como hipoplasia de carótidas o enfermedad de Moya-moya, llegando a estar presentes hasta en un 45\% según algunas series (Loddenkemper et al., 2008), por lo que todos los pacientes con AMG se les debe estudiar el SNC y su vasculatura con imágenes.

Dentro de las causas de mala agudeza visual, destacan la ambliopía y el desarrollo de desprendimiento de retina seroso (hasta en un 30\%), el cual se presenta típicamente en el área peripapilar, pudiéndose con el tiempo, extenderse a polo posterior.

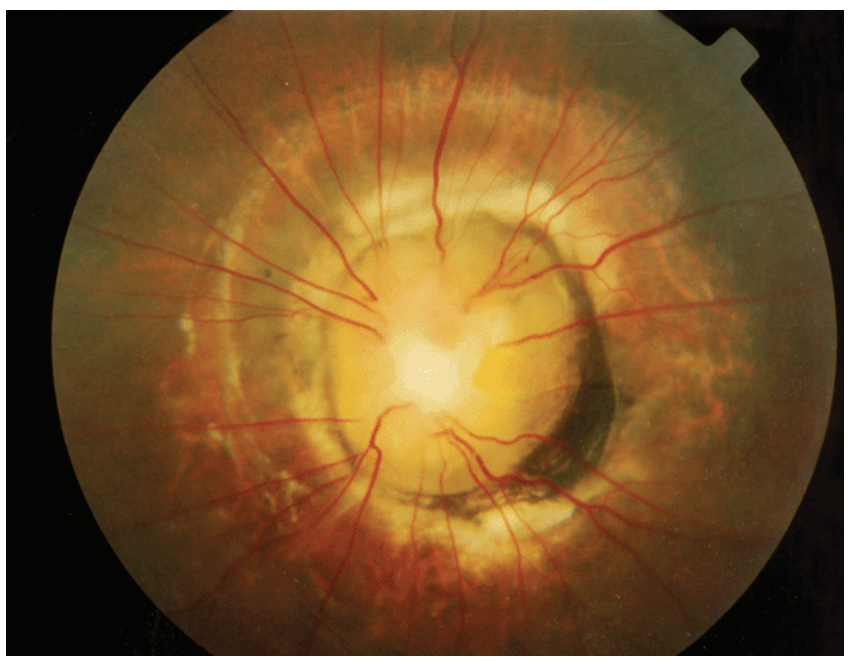

Figura 5: Papila de Morning-Glory. la emergencia radial de su vasculatura, el anillo hiperpigmentado y el tejido central glial son claves en la distinción morfológica de la papila de Morning-glory. (Pierre-Filho, 2004).
En la Tabla 1 se exponen las principales diferencias entre la AMG y el coloboma de NO. Es cierto que ambas pueden tener características comunes, pero el coloboma de NO basa su morfología en una disgenesia primaria de la fisura papilar, mientras que la AMG se genera por una dilatación anormal confinada a la papila más distal (Brodsky, 1994).

Tabla 1: Diferencias entre la papila presente en la anomalía de Morning-glory y el coloboma de NO. La excavación, el patrón de emergencia vascular y la presencia de tejido glial central son claves en la caracterización de ambas papilas.

\begin{tabular}{l|l}
\hline Anomalía Morning Glory & Coloboma de Nervio óptico \\
\hline $\begin{array}{l}\text { Nervio óptico se encuentra dentro } \\
\text { de la excavación }\end{array}$ & $\begin{array}{l}\text { Excavación se encuentra dentro del } \\
\text { nervio óptico }\end{array}$ \\
\hline Excavación central & Excavación asimétrica hacia inferior \\
\hline Tejido central glial & Sin tejido central \\
\hline $\begin{array}{l}\text { Severas alteraciones pigmentarias } \\
\text { peripapilares }\end{array}$ & $\begin{array}{l}\text { Mínima alteración pigmentaria } \\
\text { peripapilar }\end{array}$ \\
\hline
\end{tabular}

Vasculatura retinal que emerge radialmente, con vasos alterados.

Vasculatura retinal normal

\section{Estafiloma peripapilar}

El estafiloma peripapilar es una extraña afectación unilateral del nervio óptico, en donde este se aloja en una gran excavación estafilomatosa del polo posterior. En esta condición, el nervio óptico se encuentra en el fondo de la excavación, en la que además hay gran alteración del epitelio pigmentario retinal circundante, sin tejido glial central (a diferencia de la AMG) y un patrón vascular retiniano generalmente normal que dibuja el contorno de la lesión. En ocasiones, la vasculatura puede emerger radialmente, muy parecido al ocurrido en la AMG (Pollock, 1987). La agudeza visual es usualmente más pobre que las ACNO revisadas previamente y pese a lo que se pueda pensar, no todos los ojos con estafiloma peripapilar están asociados a anisometropía miópica.

El examen clínico debe ser completo debido a la prevalencia de anomalías oculares tales como: microftalmia, vitreo persistente primario hiperplásico o membrana pupilar persistente. Además, el seguimiento debe ser cercano ya que gran cantidad de pacientes pueden desarrollar complicaciones tales como cataratas o desprendimientos de retina regmatógenos (Brodsky, 1994). 

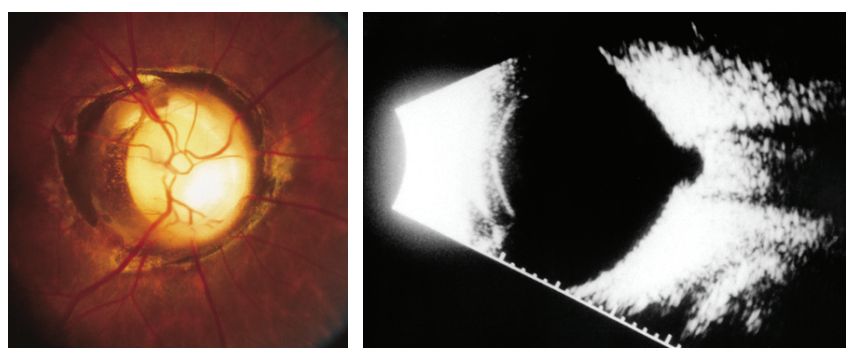

Figura 6. Estafiloma peripapilar: nervio óptico de tamaño normal que se encuentra incluido en el estafiloma, posee una vasculatura normal tanto en número como en morfología. No se aprecia tejido glial central. En la ecografía se puede evidenciar claramente el estafiloma, siendo similar a la obtenida en nuestro caso índice. (Blair, 2000)

\section{Conclusión}

Las ACNO suponen un desafío diagnóstico para el clínico debido a las características comunes y la superposición morfológica de éstas. Para llegar a un buen diagnóstico, el oftalmólogo debe realizar un examen oftalmológico completo que busque dirigidamente anomalías oculares desde los párpados hasta la retina, las cuales pueden estar presentes en los diagnósticos diferenciales y orientarnos a uno en particular. Estas características incluso pueden encontrarse en la región periauricular como ocurre en la secuencia de Goldenhar. En casos de duda diagnóstica, apoyarse en herramientas como el OCT y la ecografía puede ser útil en el estudio del nervio óptico.

Una vez realizado el diagnóstico es importante buscar las asociaciones sistémicas ya descritas para cada caso, ya que algunas de éstas incluso pueden poner en riesgo la vida de nuestro paciente, como es el caso de la enfermedad de Moya-Moya.

El manejo inicial debe estar orientado a desarrollar el mayor potencial visual de el o los ojos afectados. Nunca se debe dar por perdida la agudeza visual, y en este sentido se debe incluir siempre refracción con cicloplejia, iniciando una adecuada rehabilitación visual que incluya tratamiento de los vicios de refracción y de la ambliopía.

Se recomienda que los pacientes mantengan un control habitual, espaciado según criterio del clínico, lo que permitirá la detección temprana de complicaciones, tales como el desprendimiento de retina seroso, estrabismo o baja en la agudeza visual.

No saltarse pasos en el examen oftalmológico llevará al clínico por buen camino en el diagnóstico y seguimiento de las ACNO.

\section{Conflictos de interés}

Los autores se declaran libres de cualquier conflicto de interés.

\section{Referencias}

BeyerWB, Quencer RM \& Osher RH. (1982). Morning Glory Syndrome: A Functional Analysis Including Fluorescein Angiography, Ultrasonography, and Computerized Tomography. Ophthalmology 89, 1362-1364

Blair MP, Blair NP, Rheinstrom SD \& Jednock NJ. (2000). A Case of peripapillary Staphyloma. Arch ophthalmol 118, 1138-1139.

Brodsky MC. (1991). Septo-optic dysplasia: A reappraisal. Seminars in Ophthalmology 6, 227-232.

Brodsky MC. (1994). Congenital optic disk anomalies. Survey of Ophthalmology 9, 89-112.

Brodsky MC. (1994). Morning glory disc anomaly or optic disc coloboma. Archives of ophthalmology 112, 153 Letter.

Brodsky MC, Glasier CM, Pollock SC \& Angtuago EJC. (1990). Optic Nerve Hypoplasia: Identification by Magnetic Resonance Imaging. Archives of Ophthalmology 108, 1562-1567.

Capo H, Repka MX, Edmond JC, Drack AV, Blumenfeld L \& Siatkowski RM. (2011). Optic nerve abnormalities in children: A practical approach. Journal of AAPOS 15, 281-290.

Caprioli J \& Lesser RL. (1983). Basal encephalocele and morning glory syndrome. British Journal of Ophthalmology 67, 349-351.

De Morsier G. (1956). Etudes sur les dysraphies crânioencéphaliques. III. Agénésis du septum lucidum avec malformation du tractus optique. La dysplasie septo-optique. Schweizer Archiv Neurologie und Psychiatrie 77, 267-292.

Garcia-Filion P, Fink C, Geffner ME \& Borchert M. (2010). Optic nerve hypoplasia in North America: Are-appraisal of perinatal risk factors. Acta Ophthalmologica 88, 527-534.

Jeng-Miller (2017). Congenital anomalies of the optic disc: insights from optical coherence tomography imaging. Curr Opin Ophthalmol 28, 579-586

Jonas JB, Koniszewski G \& Naumann GOH. (1989). Morning glory syndrome (Handmann's anomaly) in congenital macrodisks. An extreme variant of "confluent optic pits". Klinische Monatsblatter fur Augenheilkunde 195, 371-374.

Kim SH, Choi MY, Yu YS \& Huh JW. (2005). Peripapillary staphyloma: Clinical features and visual outcome in 19 cases. Archives of Ophthalmology 123, 1371-1376. 
Loddenkemper T, Friedman NR, Ruggieri PM, Marcotty A, Sears J \& Traboulsi El. (2008). Pituitary stalk duplication in association with moya moya disease and bilateral morning glory disc anomaly-Broadening the clinical spectrum of midline defects. Journal of Neurology 6, 885-90.

Ophthalmic Atlas Images by EyeRounds.org, The University of lowa. 2015. Accedido en: https://webeye.ophth.uiowa.edu/eyeforum/ atlas/pages/ON-coloboma/index.htm el 05 de marzo de 2020

Pierre-Filho P de T, Limeira-Soares PH, Marcondes AM (2004). Morning glory syndrome associated with posterior pituitary Ectopia and hypopituitarism. Acta Ophthalmol Scand 82, 89-92.
Pollock S. (1987). The morning glory disc anomaly: Contractile movement, classification, and embryogenesis. Documenta Ophthalmologica 65, 439-460.

Steam Cell Care India (2019). Accedido en: https://www.stemcellcareindia.com/diseases/stem-cell-treatment-optic-nerve-hypoplasia-indial el 03 de noviembre de 2019

Taylor D. (2007). Developmental abnormalities of the optic nerve and chiasm. Eye (Lond) 21, 1271-84

Weiss AH \& Kelly JP. (2003). Acuity, ophthalmoscopy, and visually evoked potentials in the prediction of visual outcome in infants with bilateral optic nerve hypoplasia. Journal of AAPOS 7, 108-15. 